\title{
The current method for stationary mean-field games on networks
}

\author{
Diogo A. Gomes ${ }^{b}$, Diego Marcon $\sharp$, and Fatimah Al Saleh ${ }^{\natural}$
}

\begin{abstract}
We discuss first-order stationary mean-field games (MFG) on networks. These models arise in traffic and pedestrian flows. First, we address the mathematical formulation of first-order MFG on networks, including junction conditions for the Hamilton-Jacobi $(\mathbf{H J})$ equation and transmission conditions for the transport equation. Then, using the current method, we convert the MFG into a system of algebraic equations and inequalities. For critical congestion models, we show how to solve this system by linear programming.
\end{abstract}

\section{INTRODUCTION}

Mean-field games (MFG) [LL07], [HMC06], [HCM07], have been the focus of intense research. Their applications to population dynamics and pedestrian flows [LW11], [BDFMW14], crowds [BMP11], evacuation scenarios [DTT17], and traffic flows [BZP14] are of great interest. Several researchers have studied second-order MFG on networks, [CCM17], [CM16], [CCM15], [ADLT19], [LS16], [LS17]. In second-order MFGs, agents follow a controlled diffusion process and, thus, change direction infinitely often. This behavior does not occur in applications such as vehicular networks. Therefore, first-order MFGs are a natural choice for these problems. Unfortunately, prior methods are not valid for first-order MFG, where a distinct set of phenomena that includes the loss of smoothness for HamiltonJacobi (HJ) equations and lack of continuity for the value function at the vertices can occur. Because first-order MFG on networks are coupled systems of first-order MFG, we use the current method from [GNP17], [GNP16] to construct a novel approach for stationary MFG on networks.

Flows on networks have been studied intensively, see [GHP16] and [BD11]. Also, there is now a vast literature on HJ equations on networks [SC13], [CMS13], [ACCT13], [CM13], [CCM18]. Stationary first-order HJ equations on networks were examined in [SS18] and [ISM17] and the transport of measures on networks in [CDMT17]. Our MFG model can be seen as a generalization of the Wardrop equilibrium [WW52], where the routes between a target and a destination are used in such a way that travel time is independent on the choice of path. Using MFGs, we can model different travel costs and have multiple exits and entrances to the network.

\footnotetext{
$\sharp$ Universidade Federal do Rio Grande do Sul, Email: diego.marcon@ufrgs.br

b, 6 King Abdullah University of Science and Technology (KAUST), CEMSE Division, Thuwal 23955-6900. Saudi Arabia.

b Email: diogo.gomes@kaust.edu.sa

4 Mathematics and statistics department, Faculty of Science, King Faisal University, Al-Hassa, P.O. Box 400, Hofuf 31982, Saudi Arabia. Email: fatimah.saleh@kaust.edu.sa, fhalsaleh@kfu.edu.sa
}

Explicit solutions to MFG are hard to find and, in higher dimensions, apart from exceptional cases, MFGs cannot be solved algebraically. As far as the authors were aware, before this paper, there was no systematic approach to solving firstorder MFG on networks. This paper contains the following main contributions. First, we derive a model for deterministic MFG on networks that comprises incoming current of agents, edge-switching costs, and exit costs. Second, we generalize the current method to network problems and, thus, convert the MFG into a system of algebraic equations. Finally, we show how to solve the critical congestion case with linear programming methods.

\section{PRELIMINARIES}

Here, we introduce the formalism for MFG on networks. We split our discussion into statics - the network structure - and kinematics - the study of trajectories.

\section{A. Statics}

A network, $\Gamma$, is a metric space endowed with a distance $d: \Gamma \times \Gamma \rightarrow \mathbb{R}_{0}^{+}$and with the additional structure below. First, $\Gamma=\cup_{k \in \mathcal{K}} e_{k}$ where the sets $e_{k}$ are the edges and $\mathcal{K}=$ $\{1,2, \ldots, n\}$. We set $E=\left\{e_{k}: k \in \mathcal{K}\right\}$. Edges contain two distinguished points, the vertices (here we consider loopless networks; that is, edges with a single vertex are not allowed). For simplicity, we assume that any two edges intersect at a single vertex. $e_{k}$ connects two vertices, $v_{i}$ and $v_{l}$, if $v_{i}, v_{l} \in$ $e_{k}$. The set of vertices is $V=\left\{v_{i}, i \in \mathcal{I}\right\} \subset \Gamma$, where $\mathcal{I}=$ $\{1,2, \ldots, m\}$. Each edge is identified with $[0,1]$, and each vertex on that edge corresponds to 0 or 1 . More precisely, $\Gamma$ has a system of local coordinates; that is, a map $\pi:[0,1] \times$ $\mathcal{K} \rightarrow \Gamma$, such that:

1) $\pi([0,1] \times\{k\})=e_{k}$ for every $k \in \mathcal{K}$

2) Let $e_{k} \in E$. Then $\{\pi(0, k), \pi(1, k)\}=\left\{v_{i}, v_{l}\right\} \subset V$, and $\pi(\theta, k) \notin V$ for $0<\theta<1$.

3) $\pi:(0,1) \times \mathcal{K} \rightarrow \Gamma$ is injective.

The first property implies that $\pi$ is surjective, since $\Gamma=$ $\cup_{k \in \mathcal{K}} e_{k}$. Finally, for each vertex, $v_{i}$, and each edge, $e_{k}$, incident in $v_{i}$, the coordinate function, $v_{i}(k)$, gives the $x$ coordinate of $v_{i}$ in $e_{k}$; that is, $\pi\left(v_{i}(k), k\right)=v_{i}$.

Second, we address the metric, $d$, in $\Gamma$. Given $z_{1}, z_{2} \in \Gamma$,

1) For $z_{1}, z_{2} \in e_{k}$, we have $z_{1}=\pi\left(x_{1}, k\right)$ and $z_{2}=$ $\pi\left(x_{2}, k\right)$, for $x_{1}, x_{2} \in[0,1]$. Then, we set $d\left(z_{1}, z_{2}\right)=$ $\left|x_{1}-x_{2}\right|$.

2) If $z_{1}, z_{2}$ do not belong to a common edge, then

$d\left(z_{1}, z_{2}\right)=\inf \left[d\left(z_{1}, v_{i_{0}}\right)+\sum_{l=0}^{N-1} d\left(v_{i_{l}}, v_{i_{l+1}}\right)+d\left(v_{N}, z_{2}\right)\right]$

where $\left(z_{1}, v_{i_{0}}\right),\left(v_{i_{l}}, v_{i_{l+1}}\right)$, and $\left(v_{N}, z_{2}\right)$ are adjacent. 
For two points in the same edge, the distance is the distance between their coordinates; for two points on different edges, the distance is determined by the shortest path connecting the points, where each edge has length 1 .

The interior of an edge $e_{k}$ connecting $v_{i}$ with $v_{l}$, is

$$
\operatorname{int}\left(e_{k}\right)=e_{k} \backslash\left\{v_{i}, v_{l}\right\}=\pi((0,1) \times\{k\}) .
$$

$\operatorname{int}\left(e_{k}\right)$ is the topological interior of $e_{k}$. The interior of $\Gamma$ is $\operatorname{int}(\Gamma):=\bigcup_{e_{k} \in E} \operatorname{int}\left(e_{k}\right)$; note that, this does not correspond to the topological interior of $\Gamma$. The outward boundary, $\partial^{\circ} \Gamma$, is the set of vertices where agents can exit $\Gamma$, the inward boundary, $\partial^{i} \Gamma$, comprises the vertices where agents enter $\Gamma$. We assume that $\partial^{o} \Gamma \cap \partial^{i} \Gamma=\emptyset$. A function, $f: \Gamma \rightarrow \mathbb{R}$, is differentiable at $z=\pi(x, k) \in \operatorname{int}(\Gamma)$, where $x \in(0,1)$ and $k \in \mathcal{K}$, if $x \mapsto f(\pi(x, k))$ is differentiable at $x$.

\section{B. Kinematics}

Now, we examine trajectories on networks and their mathematical description. A trajectory or path on $\Gamma$, is a continuous map $\gamma:[a, b] \rightarrow \Gamma$, where $[a, b] \subseteq \mathbb{R}$. $\gamma$ is a regular trajectory if $\gamma=\pi(\mathbf{x}(t), \mathbf{k}(t))$ and

1) $\mathbf{k}:[a, b] \rightarrow \mathcal{K}$ is piecewise constant and induces a finite partition of $[a, b], a=t_{0}<t_{1}<\ldots<t_{N+1}=b$ such that $\mathbf{k}$ is constant in $\left[t_{i}, t_{i+1}\right)$.

2) $\mathbf{x}:[a, b] \rightarrow[0,1]$ is continuous at all $t \neq t_{i} \cdot \gamma$ is Lipschitz or $C^{1}$ if $\mathbf{x}$ is Lipschitz or $C^{1}$ (outside $t_{i}$ ).

The first property above excludes infinitely many transitions between edges. Also, we observe that the coordinate $\mathbf{x}(t)$ may be discontinuous at $t_{i}$ because of incompatible parameterizations at a vertex with different incident edges.

For a path, $\gamma$, the first exit time from $\Gamma$ is $T_{\partial \Gamma}:=\inf \{t \in$ $\left.[a, b] ; \gamma(t) \in \partial^{o} \Gamma\right\}$. If $\gamma$ never reaches $\partial^{o} \Gamma, T_{\partial \Gamma}=+\infty$.

\section{FIRST-ORDER MEAN-FIELD GAMES ON NETWORKS}

Now, we provide an overview of deterministic control on networks, HJ equations, and discuss the corresponding transport equations. Next, we present the MFG model and the corresponding stationary problem. Here, we consider MFG with or without congestion. In the following section, we discuss the critical congestion case, for which the corresponding MFG can be solved by linear programming.

\section{A. Calculus of variations and deterministic control}

We fix a network, $\Gamma$, where agents can move along the edges and, at the vertices, can switch to a new edge. Agents exit the network once they reach the outward boundary. We consider a running cost given by a Lagrangian $L$ : $\operatorname{int}(\Gamma) \times \mathbb{R} \times[0, T] \rightarrow \mathbb{R}, L(z, \dot{x}, t)$ or $L(x, k, \dot{x}, t)$ in local coordinates. The running cost is the cost of moving along an edge. We consider Lagrangians that depend on time; later, this dependence is given through the density of agents. Hence, the stationary problem is well defined. We assume that $L$ is smooth (in $x, \dot{x}$, and $t$ ), uniformly convex in $\dot{x}$, and bounded from below. At each vertex, $v_{i}$, agents can switch from an incident edge $e_{k}$ to another incident edge $e_{l}$ incurring in a switching cost $\psi(i, k, l)$, $\psi: V \times \mathcal{K} \times \mathcal{K} \rightarrow \mathbb{R}_{0}^{+}$. If $e_{k}$ and $e_{l}$ are not incident at $v_{i}$, we set $\psi(i, k, l)=+\infty$. Moreover, we are given a terminal cost $\phi:\left(\partial^{\circ} \Gamma \times[0, T]\right) \cup(\Gamma \times\{T\}) \rightarrow \mathbb{R}$; agents pay a terminal cost at the outward boundary at time $t \in[0, T]$ or at the terminal time $T$, if they do not leave the network.

Consider an agent who at time $t$ is at $z=\pi(x, k) \in \Gamma$. This agent seeks to minimize its cost by choosing a Lipschitz regular trajectory that minimizes

$$
\begin{aligned}
J(\gamma)= & \int_{t}^{\bar{T}} L(\mathbf{x}(s), \mathbf{k}(s), \dot{\mathbf{x}}(s), s) \mathrm{d} s \\
& +\sum_{i=1}^{N} \psi\left(\mathbf{i}\left(t_{i}^{-}\right), \mathbf{k}\left(t_{i}^{-}\right), \mathbf{l}\left(t_{i}^{+}\right)\right)+\phi(\mathbf{x}(\bar{T}), \mathbf{k}(\bar{T})),
\end{aligned}
$$

where $\left\{t_{i}\right\}$ is the partition associated to $\gamma=\pi(\mathbf{x}, \mathbf{k}), \mathbf{i}\left(t_{i}^{-}\right)$ is the vertex index at $t_{i}, \bar{T}=T_{\partial \Gamma} \wedge T$. The first term in $J(\gamma)$ is the edge travel cost, the second term the cost incurred for switching edges, and the last term the exit cost (if $T_{\partial \Gamma}<$ $+\infty$ ) or the cost at the terminal time if not leaving $\Gamma$.

Agents seek to minimize $J$. The infimum of $J$ over all Lipschitz regular trajectories with $\gamma(0)=\pi(x, k)$ is the value function $u: \Gamma \times[0, T] \rightarrow \mathbb{R}:$

$$
u(x, k, t)=\inf _{\gamma} J(\gamma)
$$

\section{B. Hamilton-Jacobi equations}

The Hamiltonian, $H$, is $H(x, k, p, t):=\sup _{\dot{x} \in \mathbb{R}}[-p \dot{x}-$ $L(x, k, \dot{x}, t)]$. If $u$ is regular, it solves the $\mathrm{HJ}$ equation

$$
-u_{t}(x, k, t)+H\left(x, k, u_{x}(x, k, t), t\right)=0,
$$

on each edge, with boundary conditions,

$$
u\left(v_{i}(k), k, t\right)=\min _{l \in \mathcal{K}}\left\{u\left(v_{i}(l), l, t\right)+\psi(i, k, l)\right\} .
$$

Theorem III.1 (Verification Theorem). Assume that $\hat{u}$ : $\Gamma \times[t, \bar{T}] \rightarrow \mathbb{R}$ is a classical solution of (III.2) in the edges of $\Gamma$, with terminal condition

$$
\hat{u}(x, k, \bar{T})=\phi(x, k)
$$

and that (III.3) holds. Then, $\hat{u}$ is the value function in (III.1).

Proof. Let $\gamma(s)=\pi(\mathbf{x}(s), \mathbf{k}(s))$ with $\gamma(t)=\pi(x, k)$. Let $t=t_{0}<t_{1}<\ldots<t_{N}<t_{N+1}=\bar{T}$ be the partition associated with $\mathbf{k}$, such that $\mathbf{k}(s)=k_{i}$ for $t_{i} \leqslant s<t_{i+1}$. By the fundamental theorem of calculus, for each $0 \leqslant i \leqslant N$,

$$
\int_{t_{i}}^{t_{i+1}} \frac{\mathrm{d}}{\mathrm{d} s} \hat{u}(\mathbf{x}(s), \mathbf{k}(s), s) \mathrm{d} s=\hat{u}\left(\mathbf{x}\left(t_{i+1}^{-}\right), k_{i}, t_{i+1}^{-}\right)-\hat{u}\left(\mathbf{x}\left(t_{i}^{+}\right), k_{i}, t_{i}^{+}\right) .
$$

Because $\frac{\mathrm{d}}{\mathrm{d} s} \hat{u}\left(\mathbf{x}, k_{i}, \cdot\right)=\hat{u}_{x}\left(\mathbf{x}, k_{i}, \cdot\right) \cdot \dot{\mathbf{x}}+\hat{u}_{s}\left(\mathbf{x}, k_{i}, \cdot\right)$, using the definition of $H$ and that $\hat{u}$ solves (III.2), we have

$$
\begin{aligned}
& \int_{t_{i}}^{t_{i+1}} L\left(\mathbf{x}, k_{i}, \dot{\mathbf{x}}, s\right) \mathrm{d} s=\int_{t_{i}}^{t_{i+1}} L+\hat{u}_{x} \dot{\mathbf{x}}+\hat{u}_{s} \mathrm{~d} s \\
& +\hat{u}\left(\mathbf{x}\left(t_{i}^{+}\right), k_{i}, t_{i}^{+}\right)-\hat{u}\left(\mathbf{x}\left(t_{i+1}^{-}\right), k_{i}, t_{i+1}^{-}\right) \\
& \geq \int_{t_{i}}^{t_{i+1}}-H\left(\mathbf{x}, k_{i}, \hat{u}_{x}, s\right)+\hat{u}_{s} \mathrm{~d} s \\
& +\hat{u}\left(\mathbf{x}\left(t_{i}^{+}\right), k_{i}, t_{i}^{+}\right)-\hat{u}\left(\mathbf{x}\left(t_{i+1}^{-}\right), k_{i}, t_{i+1}^{-}\right) \\
& =\hat{u}\left(\mathbf{x}\left(t_{i}^{+}\right), k_{i}, t_{i}^{+}\right)-\hat{u}\left(\mathbf{x}\left(t_{i+1}^{-}\right), k_{i}, t_{i+1}^{-}\right) .
\end{aligned}
$$


Note that (III.3) implies, in particular, that

$$
\hat{u}\left(\mathbf{x}\left(t_{i}^{+}\right), k_{i}, t_{i}^{+}\right)+\psi\left(k_{i-1}, k_{i}\right)-\hat{u}\left(\mathbf{x}\left(t_{i}^{-}\right), k_{i-1}, t_{i}^{-}\right) \geq 0 .
$$

These last two inequalities together with (III.4) yield

$$
\begin{aligned}
J(\gamma)= & \sum_{i=0}^{N} \int_{t_{i}}^{t_{i+1}} L\left(\mathbf{x}(s), k_{i}, \dot{\mathbf{x}}(s), s\right) \mathrm{d} s+\phi(\mathbf{x}(\bar{T}), \mathbf{k}(\bar{T})) \\
& +\sum_{i=1}^{N} \psi\left(k_{i-1}, k_{i}\right) \\
\geq & \sum_{i=0}^{N}\left[\hat{u}\left(\mathbf{x}\left(t_{i}^{+}\right), k_{i}, t_{i}^{+}\right)-\hat{u}\left(\mathbf{x}\left(t_{i+1}^{-}\right), k_{i}, t_{i+1}^{-}\right)\right] \\
& +\phi(\mathbf{x}(\bar{T}), \mathbf{k}(\bar{T}))+\sum_{i=1}^{N} \psi\left(k_{i-1}, k_{i}\right) \\
= & \hat{u}(x, k, t)-\hat{u}(\mathbf{x}(\bar{T}), \mathbf{k}(\bar{T}), \bar{T})+\phi(\mathbf{x}(\bar{T}), \mathbf{k}(\bar{T})) \\
& +\sum_{i=1}^{N}\left[\hat{u}\left(\mathbf{x}\left(t_{i}^{+}\right), k_{i}, t_{i}^{+}\right)+\psi\left(k_{i-1}, k_{i}\right)\right. \\
& \left.-\hat{u}\left(\mathbf{x}\left(t_{i}^{-}\right), k_{i-1}, t_{i}^{-}\right)\right] \geq \hat{u}(x, k, t) .
\end{aligned}
$$

Taking the infimum, we obtain $u(x, k, t) \geqslant \hat{u}(x, k, t)$.

Conversely, since $\mathcal{K}$ is finite and (III.3) holds, we construct $\tilde{\gamma}=\pi(\mathbf{x}, \mathbf{k})$, where in the edges, $\mathbf{x}$ solves

$$
\dot{\mathbf{x}}(t)=-D_{p} H\left(\mathbf{x}(t), \mathbf{k}(t), \hat{u}_{x}(\mathbf{x}(t), \mathbf{k}(t), t), t\right) .
$$

and $\mathbf{k}:[t, \bar{T}] \rightarrow \mathcal{K}$ is piecewise constant and

$$
\hat{u}\left(\mathbf{x}\left(t_{i}^{-}\right), k_{i-1}, t_{i}^{-}\right)=\hat{u}\left(\mathbf{x}\left(t_{i}^{+}\right), k_{i}, t_{i}^{+}\right)+\psi\left(k_{i-1}, k_{i}\right) .
$$

By the convexity of $L$, the preceding equation gives equality in the Legendre transform. Thus, all the inequalities in the proof with $\tilde{\gamma}$ in place of $\gamma$ are equalities. Therefore,

$$
\hat{u}(x, k, t)=J(\tilde{\gamma}) .
$$

Consequently, $\hat{u}(x, k, t) \geq u(x, k, t)$ as required.

The construction in the prior proof provides a minimizer for $J$ and, thus, an optimal strategy. The above theorem holds under weaker conditions: (III.3) can be replaced by

$$
u\left(v_{i}(k), k, t\right) \leqslant \min _{l \in \mathcal{K}}\left\{u\left(v_{i}(l), l, t\right)+\psi(i, k, l)\right\},
$$

provided that equality holds at the vextex $v_{i}$ from edge $e_{k}$ if the flow in that edge points towards the vertex.

\section{Transport equation}

Now, we derive the equation for the evolution of the population density in $\Gamma$. Let $b: \Gamma \times[0, T] \rightarrow \Gamma$ be a Lipschitz vector field that gives the dynamics in the edges; that is,

$$
\left\{\begin{array}{l}
\dot{\mathbf{x}}(t)=b(\mathbf{x}(t), \mathbf{k}(t), t), t>0 \\
\mathbf{x}(0)=x
\end{array}\right.
$$

In each edge $e_{k}$, we define the current as $j(x, k, t):=$ $b(x, k, t) m(x, k, t)$. Thus, the transport equation becomes $m_{t}(x, t)+(j(x, k, t))_{x}=0$; see, for instance, [GPV16]. We write $j=j^{+}-j^{-}$, where $j^{+} \cdot j^{-}=0$ and $j^{+}, j^{-} \geqslant 0$. At each vertex, $v_{i}$, the current from $e_{k}$ is $j(i, k, t)=$ $(-1)^{v_{i}(k)+1} j\left(v_{i}(k), k, t\right)$. The term $(-1)^{v_{i}(k)+1}$ takes into account the parameterization of the edge; a positive current is directed towards the vertex with $x=1$ and away from the vertex with $x=0$

Fix a vertex, $v_{i}$, and consider an edge $e_{k}$ incident to $v_{i}$. Suppose that the flow in $e_{k}$ transports agents towards $v_{i}$. Let $j_{k l}^{i}=j(i, k, l, t)$ denote the transition current from edge $e_{k}$ to edge $e_{l}$ at the vertex $v_{i}$. If the flow takes agents in $e_{k}$ away from $v_{i}$ or if two edges, $e_{k}$ and $e_{l}$, are not incident in $v_{i}, j_{k l}^{i}=0$. The transition currents satisfy the following

$$
j_{k l}^{i} \cdot j_{l k}^{i}=0, j_{k l}^{i}, j_{l k}^{i} \geqslant 0, k \neq l
$$

When reaching $v_{i}$, the agent switches from $e_{k}$ to $e_{l}$, where $e_{l}$ is such that $j_{k l}^{i} \neq 0$. This transition is not deterministic as different edges can have equal preference. Thus,

(i) In each edge, $e_{k}$,

$$
m_{t}(x, k, t)+(b(x, k, t) m(x, k, t))_{x}=0 .
$$

(ii) For each vertex, $v_{i}$, and every edge, $e_{l}$, incident in $v_{i}$, we have the balance conditions

$$
\sum_{k} j_{k l}^{i}=j^{\sigma}(i, l, t), \sigma=\{+,-\}
$$

Adding the equations in (III.8) at $v_{i}$ over the edges, $e_{l}$, incident in $v_{i}$, we obtain the Kirchoff law

$$
\sum_{k} j(i, k, t)=0 .
$$

When agents enter $\Gamma$ at $\partial^{i} \Gamma$, (III.8) becomes

$$
\sum_{k} j_{k l}^{i} \leqslant j^{\sigma}(i, l), \sigma=\{+,-\}
$$

together with

$$
\sum_{k} j(i, k)=-\iota_{i}
$$

where $\iota_{i}$ is the current of agents entering $\Gamma$ at $v_{i}$. These agents then leave through $\partial^{\circ} \Gamma$, where we do not impose constraints on the current since we cannot specify simultaneously the rate of agents entering the network and leaving the network. At $\partial^{o} \Gamma$, we require

$$
\sum_{k} j_{k l}^{i} \geqslant j^{\sigma}(i, l), \sigma=\{+,-\}
$$

and

$$
\sum_{k} j(i, k) \geqslant 0
$$

At an inward boundary vertex $v_{i}$, that has more than one incident edge the incoming current $\iota$ satisfies,

$$
\sum_{l} \iota_{i l}=\iota_{i}
$$

and we have,

$$
\sum_{k} j_{k l}^{i}=j^{\sigma}(i, l, t)+\iota_{i l}, \sigma=\{+,-\} .
$$




\section{Mean-field games}

As usual in MFG theory, the running cost of the agents depends on the distribution of the other agents and their actions are determined by the corresponding control problem. Accordingly, the Lagrangian, and, hence, the Hamiltonian, depends on $m$. In our model, agents seek to minimize the control problem in (III.1). The minimal cost is the value function $u(x, k, t)$. Since our agents are rational, they use the corresponding optimal strategy. Therefore, a solution of our MFG entails three quantities, the value function, $u$, for a typical agent, a distribution of agents, $m$, and the transition currents, $j_{k l}^{i}$, at the vertices $v_{i}$. In each edge $e_{k}$, we have

$$
\left\{\begin{array}{l}
-u_{t}(x, k, t)+H\left(x, k, u_{x}(x, k, t), m\right)=0 \\
m_{t}(x, k, t)-\left(m(x, k, t) D_{p} H\left(x, k, u_{x}(x, k, t), m\right)\right)_{x}=0 .
\end{array}\right.
$$

At the vertices, $v_{i}$, we require the currents and the transition currents conditions to hold. We also require the optimality condition (III.5) to hold, with equality if $-D_{p} H\left(v_{i}(k), k, u_{x}\right)(-1)^{v_{i}(k)+1} \geqslant 0$. Finally, we impose the following current transition conditions. For that, we fix a vertex, $v_{i}$, and an incident edge $e_{k}$. If $-D_{p} H\left(v_{i}(k), k, u_{x}\right)(-1)^{v_{i}(k)+1}<0$, we set $j_{k l}^{i}=0$, as in this situation, agents are departing from that edge. Otherwise, we look for $j_{k l}^{i}$ which satisfies $j_{k l}^{i} \cdot j_{l k}^{i}=0, j_{k l}^{i} \geqslant 0$ with $j_{k l}^{i}=0$, if the inequality in (III.5) is strict. Given this, we impose (III.8). At $v_{i}$, we have the complementary conditions

$$
\left\{\begin{array}{l}
j_{k l}^{i} \cdot\left(u\left(v_{i}(l), l, t\right)-u\left(v_{i}(k), k, t\right)\right)=0 \\
j_{l k}^{i} \cdot\left(u\left(v_{i}(k), k, t\right)-u\left(v_{i}(l), l, t\right)\right)=0
\end{array}\right.
$$

Given $\bar{u}\left(v_{i}\right)$ at $v_{i} \in \partial^{o} \Gamma$, with incident edge $e_{k}$, we have

$$
\left\{\begin{array}{l}
j^{-}(x, k, t)=0 \\
u\left(v_{i}(k), k, t\right) \leqslant \bar{u}\left(v_{i}\right) \\
j^{+}(x, k, t) \cdot\left(u\left(v_{i}(k), k, t\right)-\bar{u}\left(v_{i}\right)\right)=0 .
\end{array}\right.
$$

Agents may avoid an exit. Thus, we impose inequality for the value function, with equality if the exit current is positive. Then, we consider boundary conditions for $u$ at $t=T$.

In the stationary case, we consider the MFG

$$
\left\{\begin{array}{l}
H\left(x, k, u_{x}(x, k), m\right)=0 \\
-\left(m(x, k) D_{p} H\left(x, k, u_{x}(x, k), m\right)_{x}=0 .\right.
\end{array}\right.
$$

together with (III.5), (III.14) and (III.15), and the conditions for currents and transition currents from the prior subsection.

\section{CurRent method}

The current method was introduced in [GNP17] to transform one-dimensional stationary MFG with uniformly convex Hamiltonians into algebraic systems. Here, we extend this method to MFGs on networks. The key observation is that the current, $j$, is constant on edges. With this new variable, the $\mathrm{HJ}$ equation becomes algebraic. For simplicity, we work with quadratic Hamiltonians in the moment variable, $p$, but our methods are applicable to uniformly convex Hamiltonians. We set $H(x, k, p, m)=\frac{|p|^{2}}{2 m^{\alpha}}+V(x)-$ $g(m)$. Here, $V$ is a smooth function defined on $[0,1], g$ an increasing function and $0 \leqslant \alpha \leqslant 1$ is the congestion strength. $\alpha>0$ corresponds to congestion and agents face an increased cost of moving in high-density regions; that is, $L=m^{\alpha} \frac{v^{2}}{2}-V(x)+g(m)$.

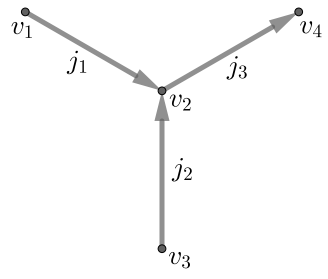

(a) Example 1 - single exit.

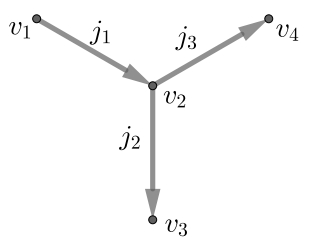

(b) Example 2 - two exits.
Fig. 1: Four-vertex networks with different boundaries.

\section{A. Current variables}

Here, we consider non-degenerate edges; that is, $j \neq 0$. In principle, we can have $j=0$, but those edges are irrelevant since no agents are crossing them. We examine the MFG

$$
\left\{\begin{array}{l}
\frac{\left|u_{x}\right|^{2}}{2 m^{\alpha}}+V(x)=g(m) \\
-\left(m^{1-\alpha} u_{x}\right)_{x}=0 .
\end{array}\right.
$$

Thus, $j=m^{1-\alpha} u_{x}$ is constant.

If $j \neq 0$, then $m \neq 0, u_{x}=j / m^{1-\alpha}$, and (IV.1) becomes

$$
\left\{\begin{array}{l}
\frac{j^{2}}{2 m^{2-\alpha}}-g(m)=-V(x) \\
m>0 \\
\int_{0}^{1} \frac{j}{m^{1-\alpha}} d x=u(1)-u(0),
\end{array}\right.
$$

If $g$ is increasing, this system has a unique solution.

\section{B. Critical congestion model}

When $\alpha=1$, (IV.1) becomes,

$$
\left\{\begin{array}{l}
\frac{\left|u_{x}\right|^{2}}{2 m}+V(x)=g(m) \\
\left(u_{x}\right)_{x}=0 .
\end{array}\right.
$$

In each edge, we have $j=u_{x}$. Then (IV.3) becomes

$$
\left\{\begin{array}{l}
\frac{j^{2}}{2 m}-g(m)=-V(x) \\
j=u(1)-u(0)
\end{array}\right.
$$

Thus, $u$ is linear in $j$ and the density, $m$, plays no role.

\section{FEASIBILITY CONDITIONS}

Now, we examine the connection between linear programming and stationary MFG. Fix a network, $\Gamma$, with outward boundary, $\partial^{\circ} \Gamma$ and exit costs, $\bar{u}\left(v_{i}\right)$, and inward boundary, $\partial^{i} \Gamma$ with incoming currents, $\iota_{i}$. The Kirchoff law (III.9) at the vertices and the constraints at $\partial^{i} \Gamma$ and $\partial^{\circ} \Gamma$, (III.11), and (III.13), are a linear feasibility problem for the current

$$
\left\{\begin{array}{l}
A j=a \\
B j \leqslant b
\end{array}\right.
$$

for suitable matrices $A, B$, and vectors $a, b$. According to (IV.2), for each feasible $j$, we recover the increment of $u$ at edges. Thus, we obtain a system of linear constraints.

$$
\left\{\begin{array}{l}
\tilde{A} u=\tilde{a} \\
\tilde{B} u \leqslant \tilde{b} .
\end{array}\right.
$$

As we illustrate next, for critical congestion, MFG can be solved by linear programming. 


\section{EXAMPLES}

Now, we study the critical case for the networks in Fig. 1.

1) Example 1: Consider the critical congestion MFG for the network in Fig. 1a. The arrows point in the direction of positive current. We set the transition cost, $\psi=0$. Given incoming currents $\iota_{1}>0$ at $v_{1}, \iota_{2}>0$ at $v_{3}$, and the exit cost $\bar{u}\left(v_{4}\right)$ at $v_{4}$, we want to find $u\left(0^{+}, 1\right), u\left(1^{-}, 1\right), u\left(0^{+}, 2\right), u\left(1^{-}, 2\right), u\left(0^{+}, 3\right), u\left(1^{-}, 3\right), j_{1}$, $j_{2}, j_{3}$, and transition currents $j_{k l}$ at $v_{2}$. We have

$$
\left\{\begin{array}{l}
u\left(1^{-}, 1\right)-u\left(0^{+}, 1\right)=j_{1}=j_{1}^{+}-j_{1}^{-} \\
u\left(1^{-}, 2\right)-u\left(0^{+}, 2\right)=j_{2}=j_{2}^{+}-j_{2}^{-} \\
u\left(1^{-}, 3\right)-u\left(0^{+}, 3\right)=j_{3}=j_{3}^{+}-j_{3}^{-} .
\end{array}\right.
$$

where $j_{i}^{+}, j_{i}^{-} \geqslant 0, j_{i}^{+} \cdot j_{i}^{-}=0$. At $\partial^{i} \Gamma=\left\{v_{i=1,2}\right\}$,

$$
j_{i}=\iota_{i}>0 \text {. }
$$

At $\partial^{\circ} \Gamma=\left\{v_{4}\right\}$, we have

$$
\left\{\begin{array}{l}
j_{3}^{-}=0 \\
u\left(1^{-}, 3\right) \leqslant \bar{u}\left(v_{4}\right) \\
j_{3}^{+} \cdot\left(u\left(1^{-}, 3\right)-\bar{u}\left(v_{4}\right)\right)=0 .
\end{array}\right.
$$

At $v_{2}$, we have the optimality conditions

$$
\left\{\begin{array}{l}
u\left(1^{-}, 1\right) \leqslant u\left(0^{+}, 3\right) \\
u\left(1^{-}, 2\right) \leqslant u\left(0^{+}, 3\right) \\
u\left(1^{-}, 1\right) \leqslant u\left(1^{-}, 2\right) \\
u\left(1^{-}, 2\right) \leqslant u\left(1^{-}, 1\right) \\
u\left(0^{+}, 3\right) \leqslant u\left(1^{-}, 1\right) \\
u\left(0^{+}, 3\right) \leqslant u\left(1^{-}, 2\right) .
\end{array}\right.
$$

(VI.4)

At $v_{2}$, we also have the complementary conditions

$$
\left\{\begin{array}{l}
j_{13} \cdot\left(u\left(0^{+}, 3\right)-u\left(1^{-}, 1\right)\right)=0 \\
j_{31} \cdot\left(u\left(1^{-}, 1\right)-u\left(0^{+}, 3\right)\right)=0 \\
j_{23} \cdot\left(u\left(0^{+}, 3\right)-u\left(1^{-}, 2\right)\right)=0 \\
j_{32} \cdot\left(u\left(1^{-}, 2\right)-u\left(0^{+}, 3\right)\right)=0 \\
j_{12} \cdot\left(u\left(1^{-}, 2\right)-u\left(1^{-}, 1\right)\right)=0 \\
j_{21} \cdot\left(u\left(1^{-}, 1\right)-u\left(1^{-}, 2\right)\right)=0
\end{array}\right.
$$

And, finally, the balance conditions

$$
\begin{aligned}
& j_{3}^{+}=j_{13}+j_{23} \\
& j_{1}^{-}=j_{31}+j_{21} \\
& j_{2}^{-}=j_{32}+j_{12} \\
& j_{3}^{-}=j_{31}+j_{32} \\
& j_{1}^{+}=j_{12}+j_{13} \\
& j_{2}^{+}=j_{21}+j_{23}
\end{aligned}
$$

SOLUTION. (VI.4) implies continuity of $u$ at $v_{2}$. Thus, $u\left(1^{-}, 1\right)=u\left(1^{-}, 2\right)=u\left(0^{+}, 3\right)$. By (VI.2), $j_{i}^{-}=0, j_{i}^{+}>0$ for $i=1,2$. Therefore, by (VI.5b) and (VI.5c), $j_{21}=j_{31}=$ $j_{12}=j_{32}=0$. Hence, by (VI.5e) and (VI.5f), $j_{13}=j_{1}^{+}>0$ and $j_{23}=j_{2}^{+}>0$. Thus, by (VI.5a), $j_{3}^{+}>0$. So, by (VI.3), $u\left(1^{-}, 3\right)=\bar{u}\left(v_{4}\right)$. Thus, using the continuity in (VI.1),

$$
\left\{\begin{array}{l}
u\left(0^{+}, 3\right)-u\left(0^{+}, 1\right)=j_{1}^{+} \\
u\left(0^{+}, 3\right)-u\left(0^{+}, 2\right)=j_{2}^{+} \\
u\left(1^{-}, 3\right)-u\left(0^{+}, 3\right)=j_{1}^{+}+j_{2}^{+},
\end{array}\right.
$$

which can be solved by standard methods.
2) Example 2: Consider the critical congestion MFG for the network in Fig. 1b. As before, we set $\psi=0$. Given $\bar{u}\left(v_{3}\right)$ at $v_{3}, \bar{u}\left(v_{4}\right)$ at $v_{4}$ and $\iota_{1}>0$ at $v_{1}$, we need to find $u\left(0^{+}, 1\right), u\left(1^{-}, 1\right)$, $u\left(0^{+}, 2\right), u\left(1^{-}, 2\right), u\left(0^{+}, 3\right), u\left(1^{-}, 3\right), j_{1}, j_{2}, j_{3} \quad$ and the transition currents $j_{k l}$ at $v_{2}$. In the edges, we have

$$
\left\{\begin{array}{l}
u\left(1^{-}, 1\right)-u\left(0^{+}, 1\right)=j_{1}=j_{1}^{+}-j_{1}^{-} \\
u\left(1^{-}, 2\right)-u\left(0^{+}, 2\right)=j_{2}=j_{2}^{+}-j_{2}^{-} \\
u\left(1^{-}, 3\right)-u\left(0^{+}, 3\right)=j_{3}=j_{3}^{+}-j_{3}^{-} .
\end{array}\right.
$$

At $\partial^{i} \Gamma=\left\{v_{1}\right\}, j_{1}=\iota_{1}>0$, and at $\partial^{o} \Gamma=\left\{v_{i=3,4}\right\}$,

$$
\left\{\begin{array}{l}
j_{i-1}^{-}=0 \\
u\left(1^{-}, i-1\right) \leqslant \bar{u}\left(v_{i}\right) \\
j_{i-1}^{+} \cdot\left(u\left(1^{-}, i-1\right)-\bar{u}\left(v_{i}\right)\right)=0 .
\end{array}\right.
$$

At $v_{2}$, we have the optimality conditions

$$
\left\{\begin{array}{l}
u\left(1^{-}, 1\right) \leqslant u\left(0^{+}, 3\right) \\
u\left(1^{-}, 1\right) \leqslant u\left(0^{+}, 2\right) \\
u\left(0^{+}, 2\right) \leqslant u\left(0^{+}, 3\right) \\
u\left(0^{+}, 2\right) \leqslant u\left(1^{-}, 1\right) \\
u\left(0^{+}, 3\right) \leqslant u\left(1^{-}, 1\right) \\
u\left(0^{+}, 3\right) \leqslant u\left(0^{+}, 2\right) .
\end{array}\right.
$$

At $v_{2}$, we also have the complementary conditions

$$
\left\{\begin{array}{l}
j_{12} \cdot\left(u\left(0^{+}, 2\right)-u\left(1^{-}, 1\right)\right)=0 \\
j_{21} \cdot\left(u\left(1^{-}, 1\right)-u\left(0^{+}, 2\right)\right)=0 \\
j_{13} \cdot\left(u\left(0^{+}, 3\right)-u\left(1^{-}, 1\right)\right)=0 \\
j_{31} \cdot\left(u\left(1^{-}, 1\right)-u\left(0^{+}, 3\right)\right)=0 \\
j_{23} \cdot\left(u\left(0^{+}, 3\right)-u\left(0^{+}, 2\right)\right)=0 \\
j_{32} \cdot\left(u\left(0^{+}, 2\right)-u\left(0^{+}, 3\right)\right)=0
\end{array}\right.
$$

and, the balance conditions

$$
\begin{aligned}
& j_{1}^{+}=j_{12}+j_{13} \\
& j_{2}^{-}=j_{21}+j_{23} \\
& j_{3}^{-}=j_{31}+j_{32} \\
& j_{1}^{-}=j_{21}+j_{31} \\
& j_{2}^{+}=j_{12}+j_{32} \\
& j_{3}^{+}=j_{13}+j_{23} .
\end{aligned}
$$

The prior conditions give the Kirchhoff law, $j_{1}=j_{2}+j_{3}$.

Solution. $u$ is continuous at $v_{2}$ by (VI.8). Because $j_{1}>$ $0, j_{1}^{-}=0, j_{1}^{+}>0$. Thus, by (VI.9d), $j_{21}=j_{31}=0$. Using (VI.9b) and (VI.9c) and using (VI.7), we get $j_{23}=j_{2}^{-}=$ $j_{32}=j_{3}^{-}=0$. Substituting in (VI.9e) and (VI.9f), we get $j_{12}=j_{2}^{+} \geqslant 0, j_{13}=j_{3}^{+} \geqslant 0$. We have four cases,

$$
\left\{\begin{array} { l } 
{ j _ { 2 } ^ { + } = 0 } \\
{ j _ { 3 } ^ { + } = 0 }
\end{array} \quad \left\{\begin{array} { l } 
{ j _ { 2 } ^ { + } > 0 } \\
{ j _ { 3 } ^ { + } > 0 }
\end{array} \quad \left\{\begin{array} { l } 
{ j _ { 2 } ^ { + } > 0 } \\
{ j _ { 3 } ^ { + } = 0 }
\end{array} \quad \left\{\begin{array}{l}
j_{2}^{+}=0 \\
j_{3}^{+}>0
\end{array}\right.\right.\right.\right.
$$

$0<j_{1}=j_{2}^{+}+j_{3}^{+}$rules out the first case. In the second case,

$$
\left\{\begin{array}{l}
u\left(1^{-}, 2\right)=\bar{u}\left(v_{3}\right) \\
u\left(1^{-}, 3\right)=\bar{u}\left(v_{4}\right)
\end{array}\right.
$$


by (VI.7). This and the continuity in (VI.6) give

$$
\left\{\begin{array}{l}
u\left(1^{-}, 1\right)-u\left(0^{+}, 1\right)=j_{1}^{+} \\
u\left(1^{-}, 2\right)-u\left(1^{-}, 1\right)=j_{2}^{+} \\
u\left(1^{-}, 3\right)-u\left(1^{-}, 1\right)=j_{1}^{+}-j_{2}^{+} .
\end{array}\right.
$$

The last two equations, (VI.10), and Kirchhoff law give $\left|\bar{u}\left(v_{3}\right)-\bar{u}\left(v_{4}\right)\right| \leqslant \iota_{1}-$ for large $\iota_{1}$, agents use both exits.

The last two cases are similar; so, we study only the third case. Accordingly, $j_{13}=j_{3}^{+}=0$ and all agents switch to $e_{2}$. By (VI.7), $u\left(1^{-}, 2\right)=\bar{u}\left(v_{3}\right)$ and $u\left(1^{-}, 3\right) \leqslant$ $\bar{u}\left(v_{4}\right)$. Using this result and the continuity, in (VI.6), we get $u\left(1^{-}, 1\right)-u\left(0^{+}, 1\right)=j_{1}^{+}, u\left(1^{-}, 2\right)-u\left(1^{-}, 1\right)=$ $j_{1}^{+}, u\left(1^{-}, 3\right)-u\left(1^{-}, 1\right)=0$. Because $j_{2}>0$, we have $u\left(1^{-}, 2\right)=\bar{u}\left(v_{3}\right)$ and $u\left(0^{+}, 2\right)=\bar{u}\left(v_{3}\right)+j_{2}$. Furthermore, $u\left(0^{+}, 3\right)=u\left(1^{-}, 3\right) \leqslant \bar{u}\left(v_{4}\right)$. Because, we must have $u\left(0^{+}, 2\right) \leqslant u\left(0^{+}, 3\right)$, we obtain the compatibility condition for this case $\bar{u}\left(v_{3}\right)-\bar{u}\left(v_{4}\right) \leqslant-\iota_{1}$. In the remaining case, the compatibility condition becomes $\bar{u}\left(v_{3}\right)-\bar{u}\left(v_{4}\right)>\iota_{1}$. In this case, one of the currents vanishes. This is natural as if one of the exit costs is too large, agents will avoid it.

\section{FINAL REMARKS}

Here, we develop the formulation of first-order MFG on networks. Using the current method, we obtain a purely algebraic system. For the critical congestion case, we can eliminate the variable $m$ in the MFG. In this case, the problem can be solved by linear programming methods. Further work is needed to understand the uniqueness of MFG on networks, improve the solvability conditions, and develop effective algorithms. Moreover, in the non-critical congestion case, we can write non-linear feasibility conditions. These are more involved but perhaps accessible to elementary tools.

Acknowledgments: This work was supported in part by King Abdullah University of Science and Technology (KAUST) baseline funds and KAUST OSR-CRG2017-3452. F. Saleh acknowledges the Deanship of Scientific Research at King Faisal University for the financial support under Nasher Track (Grant No. 186276).

\section{REFERENCES}

[ACCT13] Y. Achdou, F. Camilli, A.a Cutrì, and N. Tchou. HamiltonJacobi equations constrained on networks. NoDEA Nonlinear Differential Equations Appl., 20(3):413-445, 2013.

[ADLT19] Y. Achdou, M. Dao, O. Ley, and N. Tchou. Finite Horizon Mean Field Games on Networks. arXiv:1903.02761, 2019.

[BD11] N. Bellomo and C. Dogbe. On the modeling of traffic and crowds: A survey of models, speculations, and perspectives. SIAM review, 53(3):409-463, 2011.

[BDFMW14] M. Burger, M. Di Francesco, P. A. Markowich, and M.-T Wolfram. Mean field games with nonlinear mobilities in pedestrian dynamics. Discrete Contin. Dyn. Syst. Ser. B, 19(5):1311-1333, 2014.

[BMP11] M. Burger, P. A. Markowich, and J.-F. Pietschmann. Continuous limit of a crowd motion and herding model: analysis and numerical simulations. Kinet. Relat. Models, 4(4):10251047, 2011.

[BZP14] D. Bauso, X. Zhang, and A. Papachristodoulou. Density flow over networks: A mean-field game theoretic approach. Proceedings of the IEEE Conference on Decision and Control, 2015-February(February):3469-3474, 2014.
[CCM15] F. Camilli, E. Carlini, and C Marchi. A model problem for mean field games on networks. Discrete Contin. Dyn. Syst., 35(9):4173-4192, 2015.

[CCM17] S. Cacace, F. Camilli, and C. Marchi. A numerical method for mean field games on networks. ESAIM Math. Model. Numer. Anal., 51(1):63-88, 2017.

[CCM18] F. Camilli, E. Carlini, and C. Marchi. A flame propagation model on a network with application to a blocking problem Discrete Contin. Dyn. Syst. Ser. S, 11(5):825-843, 2018.

[CDMT17] F. Camilli, R. De Maio, and A. Tosin. Transport of measures on networks. Netw. Heterog. Media, 12(2):191-215, 2017.

[CM13] F. Camilli and C. Marchi. A comparison among various notions of viscosity solution for Hamilton-Jacobi equations on networks. J. Math. Anal. Appl., 407(1):112-118, 2013.

[CM16] F. Camilli and C. Marchi. Stationary mean field games systems defined on networks. SIAM J. Control Optim. 54(2):1085-1103, 2016

[CMS13] F. Camilli, C. Marchi, and D. Schieborn. The vanishing viscosity limit for Hamilton-Jacobi equations on networks. J. Differential Equations, 254(10):4122-4143, 2013.

[DTT17] Boualem Djehiche, Alain Tcheukam, and Hamidou Tembine. A mean-field game of evacuation in mutlilevel building. IEEE Trans. Automat. Control, 62(10):5154-5169, 2017.

[GHP16] M. Garavello, K. Han, and B. Piccoli. Models for vehicular traffic on networks, volume 9 of AIMS Series on Applied Mathematics. AIMS, Springfield, MO, 2016.

[GNP16] D. Gomes, L. Nurbekyan, and M. Prazeres. Explicit solutions of one-dimensional, first-order, stationary mean-field games with congestion. 2016 IEEE 55th Conference on Decision and Control, CDC 2016, pages 4534-4539, 2016.

[GNP17] D. Gomes, L. Nurbekyan, and M. Prazeres. One-dimensional stationary mean-field games with local coupling. Dyn. Games and Applications, 2017.

[GPV16] D. Gomes, E. Pimentel, and V. Voskanyan. Regularity theory for mean-field game systems. SpringerBriefs in Mathematics. Springer, [Cham], 2016.

[HCM07] M. Huang, P. E. Caines, and R. P. Malhamé. Largepopulation cost-coupled LQG problems with nonuniform agents: individual-mass behavior and decentralized $\epsilon$-Nash equilibria. IEEE Trans. Automat. Control, 52(9):1560-1571, 2007.

[HMC06] M. Huang, R. P. Malhamé, and P. E. Caines. Large population stochastic dynamic games: closed-loop McKean-Vlasov systems and the Nash certainty equivalence principle. Commun. Inf. Syst., 6(3):221-251, 2006.

[ISM17] R. Iturriaga and H. Sánchez Morgado. The Lax-Oleinik semigroup on graphs. Netw. Heterog. Media, 12(4):643-662, 2017.

[LL07] J.-M. Lasry and P.-L. Lions. Mean field games. Jpn. J. Math., 2(1):229-260, 2007.

[LS16] P. Lions and P. Souganidis. Viscosity solutions for junctions: well posedness and stability. Atti Accad. Naz. Lincei Rend. Lincei Mat. Appl., 27(4):535-545, 2016.

[LS17] P. Lions and P. Souganidis. Well-posedness for multidimensional junction problems with Kirchoff-type conditions. Atti Accad. Naz. Lincei Rend. Lincei Mat. Appl., 28(4):807-816, 2017.

[LW11] A. Lachapelle and M.-T. Wolfram. On a mean field game approach modeling congestion and aversion in pedestrian crowds. Transportation Research Part B: Methodological, 45:1572-1589, 2011.

[SC13] D. Schieborn and F. Camilli. Viscosity solutions of Eikonal equations on topological networks. Calc. Var. Partial Differential Equations, 46(3-4):671-686, 2013.

[SS18] A. Siconolfi and A. Sorrentino. Global results for eikonal Hamilton-Jacobi equations on networks. Anal. PDE, 11(1):171-211, 2018.

[WW52] J Wardrop and J Whitehead. Correspondence. some theoretical aspects of road traffic research. Proceedings of the Institution of Civil Engineers, 1(5):767-768, 1952. 